\title{
Rat Ultrasonic Vocalizations as Social Reinforcers-Implications for a Multilevel Model of the Cognitive Representation of Action and Rats' Social World
}

\author{
Tobias Kalenscher, Lisa-Maria Schönfeld, Sebastian Löbner, Markus Wöhr, \\ Mireille van Berkel, Maurice-Philipp Zech, and Marijn van Wingerden
}

\begin{abstract}
Rats are social animals. For example, rats exhibit mutual-reward preferences, preferring choice alternatives that yield a reward to themselves as well as to a conspecific, over alternatives that yield a reward only to themselves. We have recently hypothesized that such mutual-reward preferences might be the result of reinforcing properties of ultrasonic vocalizations (USVs) emitted by the conspecifics. USVs in rats serve as situation-dependent socio-affective signals with important communicative functions. To test this possibility, here, we trained rats to enter one of two compartments in a T-maze setting. Entering either compartment yielded identical food rewards as well as playback of pre-recorded USVs either in the 50-kHz range, which we expected to be appetitive or therefore a potential positive reinforcer, or in the $22-\mathrm{kHz}$ range predicted to be aversive and therefore a potential negative reinforcer. In three separate experimental conditions, rats chose between compartments yielding either 50-kHz USVs versus a non-ultrasonic control stimulus (condition 1), $22-\mathrm{kHz}$ USVs versus a non-ultrasonic control stimulus (condition 2), or $50-\mathrm{kHz}$ versus 22-kHz USVs (condition 3). Results show that rats exhibit a transient preference for the $50-\mathrm{kHz}$ USV playback over non-ultrasonic control stimuli, as well as an initial avoidance of 22-kHz USV relative to non-ultrasonic control stimuli on trend-level. As rats progressed within session through trials, and across sessions,
\end{abstract}

T. Kalenscher $(\varangle) \cdot$ L.-M. Schönfeld · M. van Berkel · M.-P. Zech · M. van Wingerden Comparative Psychology, Institute of Experimental Psychology, Heinrich-Heine-Universität, 40204 Düsseldorf, Germany

e-mail: Tobias.Kalenscher@hhu.de

M. van Berkel · M. van Wingerden

Social Rodent Lab, Institute of Experimental Psychology, Heinrich-Heine-Universität, 40204 Düsseldorf, Germany

\section{S. Löbner}

Institute of Linguistics and Information Science, Heinrich-Heine-Universität, 40204 Düsseldorf, Germany

M. Wöhr

Behavioral Neuroscience, Experimental and Biological Psychology, Philipps-Universität, 35032 Marburg, Germany 
these preferences diminished, in line with previous findings. These results support our hypothesis that USVs have transiently motivating reinforcing properties, putatively acquired through association processes, but also highlight that these motivating properties are context-dependent and modulatory, and might not act as primary reinforcers when presented in isolation. We conclude this article with a second part on a multilevel cognitive theory of rats' action and action learning. The "cascade" approach assumes that rats' cognitive representations of action may be multilevel. A basic physical level of action may be invested with higher levels of action that integrate emotional, motivational, and social significance. Learning in an experiment consists in the cognitive formation of multilevel action representations. Social action and interaction in particular are proposed to be cognitively modeled as multilevel. Our results have implications for understanding the structure of social cognition, and social learning, in animals and humans.

Keywords Rats · Ultrasonic vocalization - Prosocial behavior · Reinforcement learning $\cdot$ Cognitive representation $\cdot$ Multilevel categorization $\cdot$ Cascades

\section{Part I: The Experiments}

\section{Introduction}

Imagine you are passing through a heavy door that separates two parts of your university building. You notice that a person behind you also wants to walk through that heavy door. As an act of politeness, you hold the door open for him. Realizing this, he smiles at you and thanks you for your courtesy.

Why did you engage in such a (mildly) costly act of consideration? There are many putative reasons that may act in concert to support prosocial actions of this kind: adhering to the social norm that one should always help each other, following a generalized reciprocity principle as you may hope that someone else might hold a door open for you in the future, and working on your reputation as a friendly person. In addition, it is also possible that your behavior might be reinforced by the thankful response of the recipient of your help. According to this mechanism, you might have perceived the social signals emitted by him-his smile and his utterance of thankfulness - as rewarding, and, by consequence, the rewarding nature of these social signals might have increased the probability of repeating this helpful act in the future; that is, you will hold open the door for the next stranger again. This explanation is particularly intriguing as social signals are physical signals that can be multi-modally detected by the body's senses (smile: vision; words of thankfulness: auditory), yet they do not have primary hedonic value in themselves. Nevertheless, these signals have social significance that can influence, reinforce, and structure social behavior. In other words, stimuli like utterances and facial expressions can be 
understood on different levels of conceptual meaning: physical, social and motivational salience that, jointly, is perceived as part of our social world, and thus govern social behavior.

It is intuitively evident that the ability to attach motivational and emotional significance to events in the social world is of prime importance for social cognition (Fiske \& Taylor, 1984). However, our understanding of social cognition and its evolution is still incomplete. One likely reason is that we lack a proper conceptual framework to comprehend the cognitive, emotional and motivational processes associated with social stimuli. For instance, it is unclear how the attribution of motivational significance to physical stimuli is cognitively and neurally represented, and which features discriminate a social element from a non-social item. Simply speaking, individuals are influenced by social stimuli in a different way than by similar stimuli that lack social significance (e.g., a smile on the face of a display mannequin). However, it is unknown how individuals disambiguate between social and non-social stimuli, and how they attribute social and motivational significance to those stimuli.

Human social behavior is multi-faceted, is notoriously sensitive to cultural, experienced, cognitive, and gender-specific influences, and it is the outcome of a multitude of different motives. It is therefore imperative to avoid, or, at least, to control for possible confounding factors when studying social interaction. Although there is a rich literature on social cognition in the human domain (Fehr \& Fischbacher, 2003; Fehr \& Schmidt, 1999; Strombach et al., 2015), the best way to avoid confounding variations in cultural backgrounds, prior expectations and the tendency to show socially desirable behavior is to study social behavior in non-human animals. Moreover, we have recently argued in favor of complementing traditional human research with careful comparisons across species because such comparative approaches may offer answers to the question as to why humans make social and economic decisions as they do (Kalenscher \& van Wingerden, 2011). Here, we plan to use rats as model organisms. Rats are highly social animals (Blanchard \& Blanchard, 1990; Blanchard, Flannelly \& Blanchard, 1988) with a rich social behavior repertoire, including social play behavior (rough-and-tumble play; Siviy \& Panksepp, 2011; Vanderschuren, Achterberg, \& Trezza, 2016) and acoustic communication through ultrasonic vocalizations (USVs; Brudzynski, 2013; Wöhr \& Schwarting, 2013). Furthermore, rats have been shown to exhibit prosocial behavior in various contexts and ways (Ben-Ami Bartal, Decety, \& Mason, 2011; Hernandez-Lallement, van Wingerden, Marx, Srejic, \& Kalenscher, 2015; Hernandez-Lallement, van Wingerden, Schäble \& Kalenscher, 2016, 2017; Oberliessen et al., 2016; Rutte \& Taborsky, 2007).

We have recently developed a prosocial choice task (PCT; Hernandez-Lallement et al., 2015) in which actor rats made non-costly decisions yielding a reward to a partner rat, or no reward to partner, respectively (Fig. 1a). Our results have shown that actor rats developed a preference for the both-reward option, yielding a reward for both the actor and the partner, over the own-reward option, yielding a reward only to the actor, but not the partner. Remarkably, this behavior was only displayed if the partner was a real rat, but not if it was a toy rat (Fig. 1b, c). The extent of prosocial behavior was not uniform across the animals; there was large individual variability between rats in their mutual-reward preference levels, as indicated by the 

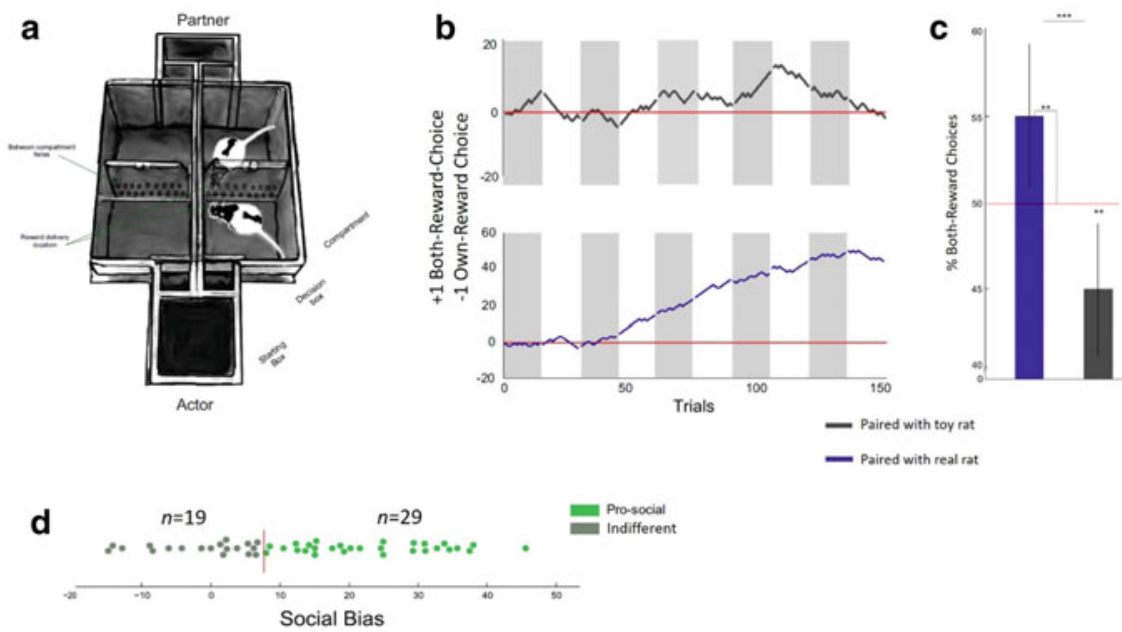

Fig. 1 Prosocial choice task. a Double T-maze apparatus for quantifying mutual-reward preferences in pairs of rats. The actor rat chooses to enter either a both-reward compartment (both rats receive identical food rewards), or an own-reward compartment (only the actor receives a reward, but not the partner). The partner is always directed towards the opposite compartment facing the actor. Actor's and partner's compartments are separated by a transparent, perforated wall, allowing rats to see, hear and smell each other. b Example choice of one rat. The tally is increased by 1 every trial the actor rats makes a both-reward choice, and decreased by 1 every trial the actor rat makes an ownreward choice. Upper panel: actor rat paired with a toy rat. Lower panel: same actor rat paired with a real partner rat. c Mean percentage of both-reward choices, averaged across all rats and sessions. d Social bias scores. For each rat, the social bias score represents the percent differences in bothreward choices between the social and toy conditions. The social bias score can be interpreted as the added value of both-reward outcomes. The vertical bar represents the upper $95 \%$ confidence interval limit, which was based to categorize rats as prosocial (green dots; social bias scores exceeding the upper confidence interval limit), and indifferent (grey dots; social bias scores within the confidence interval limits) $* * \mathrm{p}<0.05 ; * * * \mathrm{p}<0.001$. Adapted from Hernandez-Lallement et al. (2015)

wide distribution of social bias scores (Fig. 1d; the social bias scores represent the percent differences in both-reward choices between the social and toy conditions and can be interpreted as the added value of both-reward outcomes).

In a follow-up lesion study, we found that mutual-reward preferences in rats disappeared after lesions of the basolateral amygdala (Hernandez-Lallement et al., 2016), a brain structure implicated in emotional processes (LeDoux, 1994; LeDoux, Cicchetti, Xagoraris, \& Romanski, 1990) as well as social and non-social reward representation (Chang et al., 2015; Janak \& Tye, 2015). Our results showed that the social bias score, indicating the added value placed on mutual reward outcomes, turned negative in amygdala-lesioned rats (Fig. 2a) because they chose the bothreward option less often when paired with a real rat than when paired with a toy. This suggests that, in contrast to sham-lesioned animals, amygdala-lesioned rats 

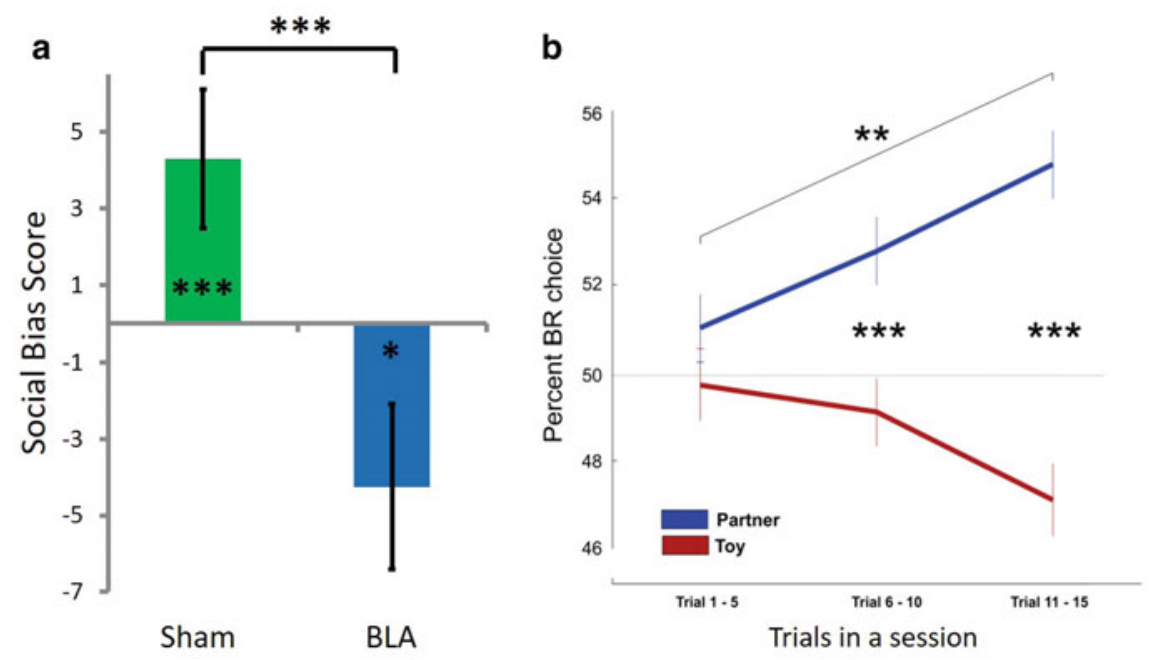

Fig. 2 Social reinforcement learning in rats is amygdala-dependent. a Lesions to the basolateral amygdala (BLA) abolish mutual-reward preferences in rats, as indicated by negative social bias scores. Adapted from (Hernandez-Lallement et al., 2016). b Rats re-acquire both-reward (BR) preferences across trials in sessions after the compartment-contingency assignment was reversed. Adapted from (Hernandez-Lallement, van Wingerden, et al., 2017). *p $<0.05 ; * * \mathrm{p}<0.01 ; * * * \mathrm{p}<$ 0.001

failed to attach positive value to rewards delivered to partners; hence, the amygdalalesioned animals behaved as if they had turned callous to the welfare of other rats (Hernandez-Lallement, van Wingerden, \& Kalenscher, et al., 2017).

To better understand the emergence of mutual-reward preferences in non-lesioned control rats, we exploited the fact that the task contingencies were frequently reversed because the both-reward assignment to one of the two actor compartments was pseudo-randomized across testing days and rats (Hernandez-Lallement, van Wingerden, et al., 2017). We found that both-reward choices were at chance level in the first few trials after a contingency reversal, but gradually increased across trials (Fig. 2b). This finding suggests that rats re-learn which compartment yields reward to both rats after every contingency change. We hypothesized that such re-learning can be explained by standard reinforcement learning mechanisms (Sutton \& Barto, 2012), with one notable exception. Because the payoff to the actor rat is always identical after own-reward or both-reward choices and in the partner- and the toyconditions, and because the only difference between conditions is the social context, the reinforcer must be of social nature. Two non-mutually-exclusive mechanisms are conceivable by which social signals, whatever they are, may reinforce mutual-reward choices (Hernandez-Lallement, van Wingerden, et al., 2017): partner rats might emit social signals upon reward receipt that are rewarding to the actor rats, reinforcing the actor's behavior that yielded reward to the partner. In addition, missing out on reward might prompt the emission of distress or complaint signals by the partner that 
are aversive to the actor rats, resulting in the avoidance of behaviors associated with these aversive complaint signals.

To date, it is unknown what kind of signals might serve as social reinforcers. However, several lines of evidence suggest that putative candidate signals for appetitive and aversive social reinforcement are rat USVs. Rats emit USVs in the 50$\mathrm{kHz}$ range in positive affective states, for example, during rough-and-tumble play (Knutson, Burgdorf, \& Panksepp, 1998; Lukas \& Wöhr, 2015), tickling (Ishiyama \& Brecht, 2016; Panksepp \& Burgdorf, 2000), or after amphetamine injections (Burgdorf, Knutson, Panksepp, \& Ikemoto, 2001; Engelhardt, Fuchs, Schwarting, $\&$ Wöhr, 2017). By contrast, rats vocalize in the $22-\mathrm{kHz}$ range in negative affective states, e.g., during threatening situations or fear conditioning (Brudzynski \& Ociepa, 1992; Calvino, Besson, Boehrer, \& Depaulis, 1996; Parsana, Li, \& Brown, 2012; Sales, 1972).

Rats show a strong, but short-lived orientation response and transient social approach behavior towards playback of pre-recorded 50-kHz USVs as well as avoidance of 22-kHz USV playback (Wöhr \& Schwarting, 2007), and will perform more instrumental actions to obtain $50 \mathrm{kHz}$ than $22 \mathrm{kHz}$ USV playback (Burgdorf et al., 2008). Moreover, 50-kHz USV playback (Willuhn et al., 2014) or observing another rat getting rewarded (Kashtelyan, Lichtenberg, Chen, Cheer, \& Roesch, 2014) elicits dopamine release in the nucleus accumbens, one of the key brain mechanisms for reinforcement learning (Parkinson, Robbins, \& Everitt, 1996). In addition, 50- and 22-kHz signals elicit increases, or decreases respectively, in tonic firing activity in single neurons in the rat amygdala (Parsana et al., 2012), the very same brain structure whose integrity is necessary for expressing mutual-reward preferences in our PCT (Fig. 2a; Hernandez-Lallement, van Wingerden, \& Kalenscher, 2017; Hernandez-Lallement et al., 2016).

Taken together, this evidence is in line with the hypothesis that $50-$ and $22-\mathrm{kHz}$ USVs might serve as candidate signals for appetitive social reinforcement, or aversive social reinforcement respectively. Moreover, rats engaged in the PCT indeed vocalize, both in the 22 and $50 \mathrm{kHz}$ domain (unpublished observations). We thus set out to investigate whether the playback of pre-recorded USVs in the context of the prosocial choice task setup would be as effective in driving choice behavior as the putative social signals emitted by partner rats in the full version of the PCT, while keeping task contingencies as close to the original PCT as possible. Specifically, we hypothesized that $50-\mathrm{kHz}$ USV stimuli induce approach behavior and, thus, enhanced preference for outcomes associated with playback of $50-\mathrm{kHz}$ USV playbacks. We furthermore hypothesized that $22-\mathrm{kHz}$ USV stimuli are avoided by the rats, resulting in decreased preference for $22-\mathrm{kHz}$ USV outcomes. In the following, we will present evidence that USVs, in contrast to similar acoustic stimuli of non-social nature, indeed have transient motivating properties and can drive spatial preferences linked to social outcomes as observed in the PCT.

Importantly, we go one step further than merely evaluating the social reinforcement hypothesis (Hernandez-Lallement, van Wingerden, et al., 2017). This hypothesis is useful in describing the cognitive mechanisms underlying mutual-reward preferences, but leaves open the question how rats cognitively construe a social situation 
characterized by the presence of conspecifics and/or USVs. More specifically, it is unclear how a rat conceptually links and represents the several stimulus levels-the USV's physical dimension (rhythmic oscillations of air compression and deflation), their emotional level (the putative enjoyment or aversiveness of listening to USVs) and their motivational level $(50-\mathrm{kHz}$ USVs are wanted and prompt action to obtain them, 22-kHz USVs are avoided and prompt action to evade them) - into a coherent cognitive representation of a social situation. A promising approach to understand how rats cognitively construct their social world needs to transcend beyond the limitations of traditional reinforcement learning theory, and enter the realm of philosophy. Therefore, in addition to presenting evidence that rats attribute incentive value to USV playback, we will conclude this article with a theoretical perspective, inspired by linguistic theory, on the rat's cognitive representation of its social world. This theory addresses the point of multilevel cognitive representation of a social act, and how this can guide learning about social interaction.

\section{Methods}

\subsection{Subjects}

The experiment was approved by German authorities (Landesamt für Natur, Umwelt und Verbraucherschutz) and conducted according to the European Union Directive 2017/63/EU. Fifteen male Long-Evans rats (Charles River Laboratories, Calco, Italy) were housed in groups of three and kept under a reversed $12 \mathrm{~h}$-dark/light cycle (lights off at $7 \mathrm{am}$ ). The housing room was at a constant temperature of $20 \pm 2{ }^{\circ} \mathrm{C}$ and a humidity of $60 \%$. Rats received standard rodent laboratory food (Sniff, Soest, Germany), and water ad libitum. At the start of the experiment, food access was restricted to keep the animals at $90 \%$ of their free feeding body weight. Animals were randomly assigned to one of two groups differing in the stimulus material (see acoustic stimuli below): $\mathrm{USV}_{\text {Type-1 }}(\mathrm{n}=7)$ and $\mathrm{USV}_{\text {Type-2 }}(\mathrm{n}=8)$.

\subsection{Experimental Setup}

The playback experiment aimed to evaluate the effectiveness of playback of prerecorded USVs in shaping spatial preferences as observed in the PCT (HernandezLallement et al., 2015). As such, we employed the same behavioral setup as in the PCT, but with the following minor modifications. Each side of the maze (front: actor side; back: partner side) consisted of a start box measuring $31 \times 20 \times 40 \mathrm{~cm}$ leading via two doors to separate choice compartments, measuring $30 \times 30 \times 40 \mathrm{~cm}$ (Fig. 1a). Thereby, two pairs of facing actor-partner compartments were created (left and right sides). The outer walls of the maze and the doors leading to the choice compartments 
were opaque whereas the choice compartments themselves were separated from each other and from the opposite half of the maze by translucent walls containing an aluminum grid (approximately $80 \%$ open) in the lower half to facilitate sound transmission from the partner to the actor side, or vice versa. Instead of a social partner, in this jukebox experiment, ultrasonic speakers (Ultrasonic Dynamic Speaker Vifa, Avisoft Bioacoustics, Germany) were placed in each partner compartment to deliver acoustic stimuli at the vertical level of the actor animal's head at a distance of about $10 \mathrm{~cm}$ from the grid wall. As in the PCT, food rewards consisting of three sucrose pellets (45 mg dustless precision pellets, Bio-Serv, Germany) were delivered though a funnel into the choice compartment after playback of the acoustic stimuli.

\subsection{Acoustic Stimuli}

Three different types of acoustic stimuli were presented: $50-\mathrm{kHz}$ USV stimuli, 22$\mathrm{kHz}$ USV stimuli and background noise corresponding to the respective USV stimuli. All stimuli were presented with a sampling rate of $192 \mathrm{kHz}$ in a 16-bit format for $5 \mathrm{~s}$.

To determine whether the rat strain used to generate USVs mattered, or generalized across strains, we used two different sources of USV stimuli: type-1 stimuli were USVs recorded from Wistar rats, and described in detail by Wöhr and Schwarting (2007) and Sadananda, Wöhr \& Schwarting (2008). Type-2 stimuli were based on calls recorded from pairs of interacting male Long-Evans rats. In brief, type-1 50$\mathrm{kHz}$ USVs were recorded from a male Wistar rat exploring a cage containing scent from a cage mate. The stimulus consisted of 19 calls (total calling time: $1.19 \mathrm{~s}$ ). Fourteen of these calls were frequency-modulated and five were flat. Call duration was $0.06 \pm 0.01 \mathrm{~s}$ (mean $\pm \mathrm{SEM}$ ); peak frequency: $61.41 \pm 1.51 \mathrm{kHz}$; bandwidth: $5.06 \pm 1.09 \mathrm{kHz}$. The type- $250-\mathrm{kHz}$ calls were recorded during investigation of an unfamiliar juvenile conspecific by an adolescent rat. The stimulus consisted of 15 calls (total calling time: $1.47 \mathrm{~s}$ ). Eleven of these calls were frequency-modulated and four were flat. Call duration was $0.10 \pm 0.02 \mathrm{~s}$ (mean \pm SEM); peak frequency: $51.63 \pm 1.14 \mathrm{kHz}$; bandwidth: $6.09 \pm 1.35 \mathrm{kHz}$. Eighteen different 50-kHz USV stimuli were generated by randomizing the order of the individual calls using SASLab Pro (version 5.2.08, Avisoft Bioacoustics, Glienicke, Germany). Background noise stimuli corresponding to the $50-\mathrm{kHz}$ USV stimuli were generated by applying a band-rejection filter to eliminate the calls in the USV stimuli, leaving only background noise. The filter was set as to remove all signal components between 20.90 and $80.00 \mathrm{kHz}$. 50-kHz USV stimuli were played at approximately $69 \mathrm{~dB}$ and corresponding background noise was played at approximately $42 \mathrm{~dB}$ (measured from a distance of about $10 \mathrm{~cm}$ ). Type- $122 \mathrm{kHz}$ calls were recorded from a male Wistar rat after applications of foot-shocks. Call duration was $1.18 \pm 0.06 \mathrm{~s}$; peak frequency: $23.61 \pm 0.07 \mathrm{kHz}$; bandwidth: $1.37 \pm 0.05 \mathrm{kHz}$; type-2 22-kHz stimuli consisted of calls from another male adolescent Long-Evans rat investigating an unfamiliar juvenile conspecific. Eighteen USV stimuli with a duration of $5 \mathrm{~s}$ were generated by randomizing the order of 4 calls. The average duration of the calls was $0.80 \pm$ 
$0.07 \mathrm{~s}$ (mean $\pm \mathrm{SEM}$ ) with a peak frequency of $26.30 \pm 0.02 \mathrm{kHz}$. Creation of corresponding background noise was similar to the $50-\mathrm{kHz}$ stimuli, only now all signal components between 21.40 and $68.30 \mathrm{kHz}$ and between 69.80 and $100.00 \mathrm{kHz}$ were removed. The playback loudness was adjusted so that the ultrasonic components in the 22-kHz USV stimuli were played at approximately $69 \mathrm{~dB}$. As such, the loudness of the background noise component was at approximately $32 \mathrm{~dB}$.

\subsection{Task Design}

Behavioral tests were performed under red light during the active period of the rats on consecutive weekdays. Before the beginning of the experiment, all rats received one day of habituation to the maze and 14 days of shaping sessions where they were gradually introduced to the testing conditions. Shaping procedures were similar to PCT training and consisted of daily sessions where animals acquired the trial structure (doors opening, compartment choice, doors closing, pellet delivery and consumption) up until the point where behavioral training was similar to the final test procedure except that no acoustic stimuli were presented.

In the final task, rats chose to enter one of the two choice compartments. Entering resulted in acoustic playback for five seconds. This $5 \mathrm{~s}$ USV playback period corresponds to the trial stage in the PCT when the partner is directed to the compartment facing the choice compartment with the actor animal, when the animals can interact acoustically through the aluminum grid. Ultimately, a food reward (three food pellets) was delivered to the actor rat, independent of which compartment was entered.

All rats performed the task under three conditions (Fig. 3), each for 8 consecutive sessions. Under condition 1 (50-vs-noise), a 50-kHz USV stimulus was played back in the choice compartment on one side and corresponding background noise in the choice compartment on the other side. Condition 2 (22-vs-noise) was identical to condition 1, except that a $22-\mathrm{kHz}$ USV stimulus was presented together with corresponding background noise. In condition 3 (50-vs-22), the $50-\mathrm{kHz}$ stimulus was played in one choice compartment and the $22-\mathrm{kHz}$ stimulus was played in the other choice compartment. The order of experimental conditions was pseudo-randomized across rats within the groups, and the USV-compartment assignment was pseudorandomized across days, ensuring that a given USV stimulus was not assigned to one side for longer than two days in a row. This pseudo-randomization approach was employed to mimic the pseudo-random assignment of the Both Reward option over days in the PCT, and to disambiguate playback preferences from potential side biases and habit development.

Each condition encompassed 8 daily testing sessions, which in turn consisted of four forced trials and 16 free trials. In the forced trials, only one door was opened in a pseudo-randomized order to allow rats to sample and learn the current assignment of acoustic stimuli to the choice compartments. In the free trials, both doors were opened at the same time and rats were able to choose which side to enter. Data is only reported for the free trials. 


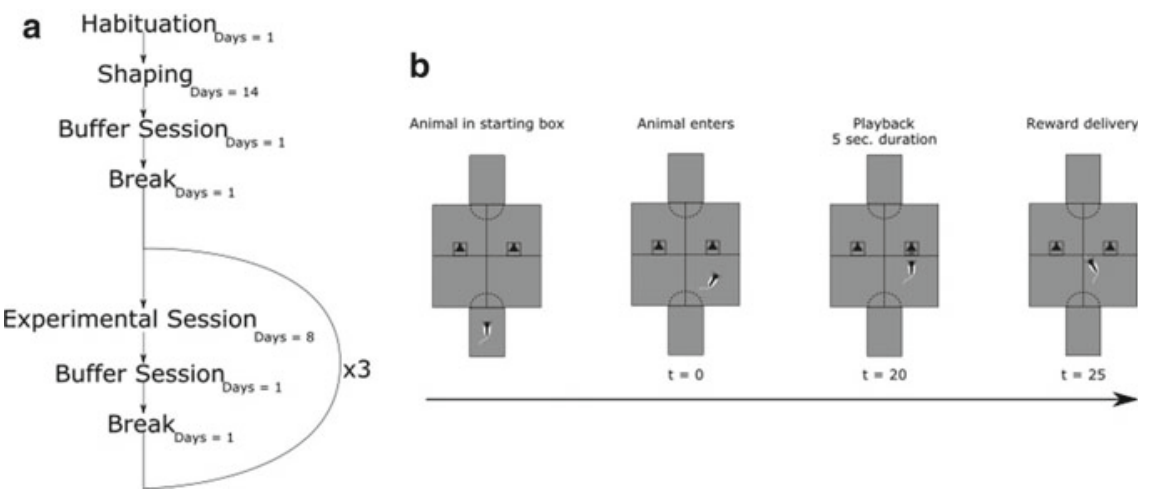

Fig. 3 Sequence of the training and testing procedure and an individual experimental trial. a All animals went through habituation to the maze (Days $=1$ ) and shaping (Days $=14$ ), where they were gradually familiarized with the testing conditions. Afterwards, a buffer session (Days $=1$; identical to the last shaping condition) took place, followed by a one-day break (Days $=1$ ). Subsequently, rats were trained and tested in the final task (Days $=8$ ), again followed by a buffer session (Days = 1 ) and a break (Days $=1)$. The procedure for the experimental sessions was repeated for all three conditions (curved arrow). b Before the beginning of a new trial, the animal was placed in the start box. Either one door (forced trials) or both doors (free trials) were opened, and, once the animal entered one of the two compartments, doors were closed and the trial timer was started $(t=0 \mathrm{~s})$. After a delay of twenty seconds $(t=20 \mathrm{~s})$, the USV stimulus was played back for five seconds in the respective compartment. Twenty-five seconds after trial onset $(t=25 \mathrm{~s})$, the food reward was delivered. After reward consumption rats were put back into their starting boxes for the next trial

Figure $3 \mathrm{~b}$ shows the sequence of an individual trial. In each trial, the animal is placed in the start box and the two doors leading to the choice compartments are opened. Once the animal enters one compartment, the doors are closed and the trial starts. After a delay of $20 \mathrm{~s}$, the acoustic stimulus is played for $5 \mathrm{~s}$. Subsequently, the food reward is delivered. After reward consumption, the animal is placed back into the start box for the next trial to begin. Adherence to the time points during each trial was ensured by a custom-made software script (Matlab 2014b, MathWorks Inc., USA) that also initiated the playback of acoustic stimuli (Avisoft-recorder, Avisoft Bioacoustics, Germany). After a session was finished, the maze was cleaned with a $70 \%$ ethanol solution to remove dirt and odor cues.

Both groups of rats, group $1\left(\mathrm{USV}_{\text {Type-1 }}\right)$ and group $2\left(\mathrm{USV}_{\text {Type-2}}\right)$ performed this task; as described above, the only difference between the groups was the origin of the acoustic stimuli.

\subsection{Data Analysis}

Anticipating a transient response to the USV stimuli (Wöhr \& Schwarting, 2012), we took advantage of the expected decay in preference both within and across sessions by 
using a cluster-based permutation test derived from EEG/MEG/LFP time-frequency and spatio-temporal analysis included in the FieldTrip analysis Toolbox (Oostenveld et al. 2011). Briefly, in cluster permutation analysis, voxels (in our case, units of session-trial such as for example S3-T4) are assessed for significance by comparing the playback preference (choices of USV) across rats for that session-trial combination to a randomly permuted $(\mathrm{N}=1000$ times) choice matrix (shuffling the position of USV choices but not the proportion). A reference distribution of preferences scores was constructed by averaging across rats for each session-trial unit across the preference scores resulting from the randomly permutated datasets and collection of these averages. Units of session-trial in the original dataset were flagged as significant if they fell outside the $99 \%$ confidence interval of this reference distribution. Clustering then took place by including adjacent significant units in a larger cluster (criterion: next-door-neighbours in horizontal (trial) or vertical (session) dimensions). The cluster statistic that resolves the multiple-comparison problem is computed by comparing the summed preferences for this cluster with the highest preference-sum of any cluster generated per random iteration (i.e. 1000 max-sum clusters). If, for positive (negative) clusters, the summed cluster score is higher (lower) than the $2.5 \%$ tail of the random cluster scores, the cluster as a whole is flagged as significant.

Following the analysis convention established for the PCT by HernandezLallement, van Wingerden, et al. (2017), we also subdivided each session into three blocks of five trials and computed the mean compartment preference across trials within each block to contrast preferences between blocks. Analyses were performed using Matlab (2014b, MathWorks Inc., USA).

\section{Results}

As expected from the USV playback literature, we found a transient preference for the $50 \mathrm{kHz}$ playback in the 50-vs-noise condition (Fig. 4a) and a transient preference against the $22 \mathrm{kHz}$ playback in the 22-vs-noise condition (Fig. 4b). Clusterpermutation analysis indicated a significant $2 \times 2$ cluster spanning sessions 1-2 $\times$ trials $1-2$ ( $\mathrm{p}<0.05$ cluster permutation test, outlined in a white rectangle) in favor of the $50 \mathrm{kHzUSVs}$ in the 50-vs-noise condition, while the transient preference against the $22-\mathrm{kHz}$ playback in the 22 -vs-noise condition visible in early trials across sessions did not reach statistical significance. Surprisingly, the sessions offering a direct choice between $50-$ and $22-\mathrm{kHz}$ USV stimuli did not replicate this pattern. Instead of exhibiting a clear preference, rats were mostly indifferent between the 50and 22-kHz USV playback (Fig. 4c), suggesting the possibility of an interaction of the call types when presented in the same setting.

This observation was supported by a more standard analysis, confirming that rats chose the compartment associated with USV stimulation significantly more often in the 50-vs-noise than the 22-vs-noise condition with both stimulus classes (pairedsample t-test, $t_{(14)}=2.16$, p $<0.05$; Fig. 5a). We observed inter-individual differences between the preference strengths for $50-\mathrm{kHz}$ USVs $(50-\mathrm{kHz}$ vs. control) and $22-\mathrm{kHz}$ 
a

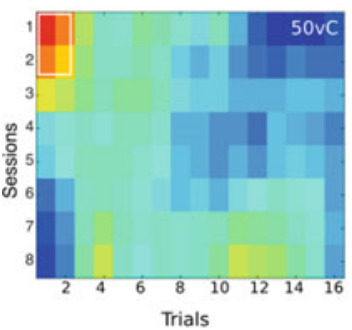

b

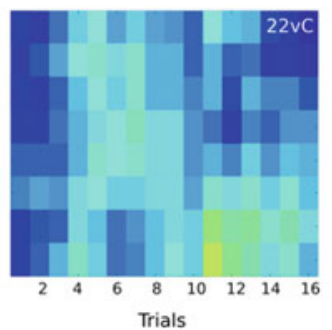

c

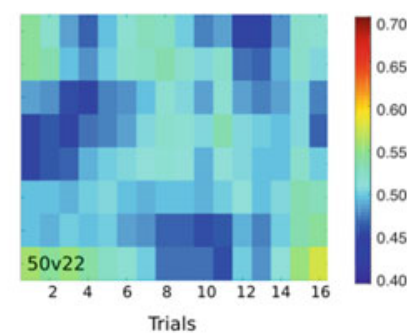

Fig. 4 Preference maps for the three conditions, calculated for each session-trial unit, averaged across rats and smoothed using a 3-unit kernel. PseudoColor scale indicates level of preference for stimulus A (hot colors) vs stimulus B (cool colors). a $50 \mathrm{kHzUSV}$ versus control, b $22 \mathrm{kHz}$ USV versus control, c $50 \mathrm{kHz}$ versus $22 \mathrm{kHz}$ USV. White rectangle: significant preference cluster ( $\mathrm{p}<$ 0.05 cluster permutation test, corrected for multiple comparisons)
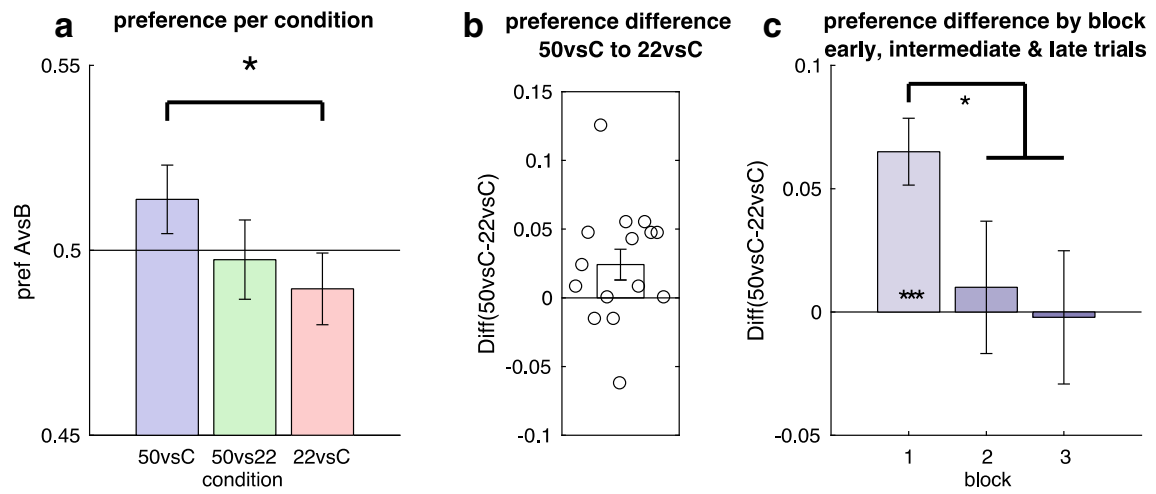

Fig. 5 Preference difference for 50- over 22-kHz USV s when paired with its control stimulus, a preference per condition considering both stimuli types, all sessions and all trials, $\mathbf{b}$ difference in preference considering both stimuli types, all sessions and all trials. Barplots indicate mean difference in preference for the $50-\mathrm{kHz}$ versus Noise minus preference for $22-\mathrm{kHz}$ versus Noise, \pm SEM. Dots represent individual rats. $\mathbf{c}$ Same as in $\mathbf{b}$, but now broken up in three blocks of five trials (trials $1-5,6-10$ and $11-16$ )

USVs (22-kHz vs. control; Fig. 5b). Blockwise-analysis, grouping trials 1-5, 6-10 and 11-16, showed that, in line with previous reports (Seffer, Schwarting, \& Wöhr, 2014; Willuhn et al., 2014; Wöhr \& Schwarting, 2007, 2012), the difference between the playback conditions was especially pronounced in the first block of five trials ( 6.5 $\pm 1.4 \%$, tr. $1-5$, one-sample t-test vs. $0 ; t_{(14)}=4.80 ; \mathrm{p}<0.001$, Fig. $\left.5 \mathrm{c}\right)$, as compared to blocks $2\left(1.0 \pm 2.7 \%\right.$, tr. $6-10 ; t_{(14)}=0.37$; n.s. $)$ and $3(-0.2 \pm 2.2 \%$, tr. $11-16$; $t_{(14)}=-0.10 ;$ n.s.). Indeed, the difference in preference in block 1 was significantly larger than the preference differences of blocks $2-3$ combined (paired-sample t-test; $t_{(14)}=2.88 ; \mathrm{p}=0.01$ ), confirming the transient nature of the effectiveness of USV playback in influencing spatial preferences. 
Such a pattern of results could stem from either a preference for 50-kHz USVs over control stimuli, an avoidance of $22-\mathrm{kHz}$ USVs over control stimuli, or both. Comparing the preference in the first block to the rest of the session suggests that only the preference for $50-\mathrm{kHz}$ USVs over control was significantly higher in the first block ( 53.8 vs. $\left.50.5 \% ; t_{(14)}=2.41 ; \mathrm{p}<0.05\right)$ while no differences could be detected in the $22-\mathrm{kHz}$ USVs vs control condition (47.3 vs. $49.8 \% ; t_{(14)}=-1.14$; n.s.).

To gain further insights into the temporal pattern of the preference habituation effects and directly compare the effects found through the cluster based permutation approach, we compared preference for compartments in first trial block of the first half of sessions (sessions 1-4) with preferences in the second half of sessions (sessions 5-8). Interestingly, though some attenuation in preference across sessions could be found, the preference difference between the 50-vs-Noise and 22-vs-Noise condition for the first block showed up in the first half $\left(6.7 \pm 2.8 \%, t_{(14)}=2.43 ; \mathrm{p}<0.05\right)$ and the second half $\left(6.3 \pm 2.7 \%, t_{(14)}=2.35 ; \mathrm{p}<0.05\right)$ of sessions. However, only in the first half of the sessions did the preference in the first block of trials differ significantly from indifference in the 50 versus control condition $\left(55.3 \pm 1.9 \%, t_{(14)}\right.$ $=2.78, \mathrm{p}=0.01$, Fig. 6a).

Taken together, these results confirm that rats exhibit a transient preference for playback of 50-kHz USVs over non-ultrasonic control stimuli, combined with a trend towards avoidance of 22-kHz USV playback. As such, it seems plausible that USVs could be one channel of social feedback involved in driving spatial preferences linked to social outcomes in the PCT.

Finally, we asked if our Long-Evans rats responded differently to USVs originating from Long-Evans conspecifics (USV type-2 calls), or from rats from a different strain (Wistar rats; USV type-1 calls). However, our results showed that the pattern of results did not significantly differ between the USV-types used (Fig. 6b, independent samples t-tests at the level of $50 \mathrm{kHz}$ playback, $22 \mathrm{kHz}$ playback or the difference; all $\left|t_{(13)}\right|<0.25$; all $\left.\mathrm{p}>0.05\right)$, suggesting that there is no evidence that rats discriminate between the strains of the USV sources.

\section{Discussion}

In this article, we present evidence supporting our hypothesis that USVs could act as social reinforcers, driving spatial preferences as observed in the pro-social choice task. In line with the social reinforcement hypothesis (Hernandez-Lallement, van Wingerden, et al., 2017), we theorized that USVs reinforce behavior that is associated with USV playback, but acoustic stimuli in a similar frequency range, yet without the social significance of USVs, do not act as social reinforcers. More specifically, we expected that $50-\mathrm{kHz}$ USVs act as positive reinforcers, and that the probability of repeating actions coupled to $50-\mathrm{kHz}$ USVs playback is larger than the probability of repeating actions associated with 22-kHz USVs or a non-ultrasonic control stimulus (Burgdorf et al., 2008). By contrast, we predicted that 22-kHz USVs act as negative 


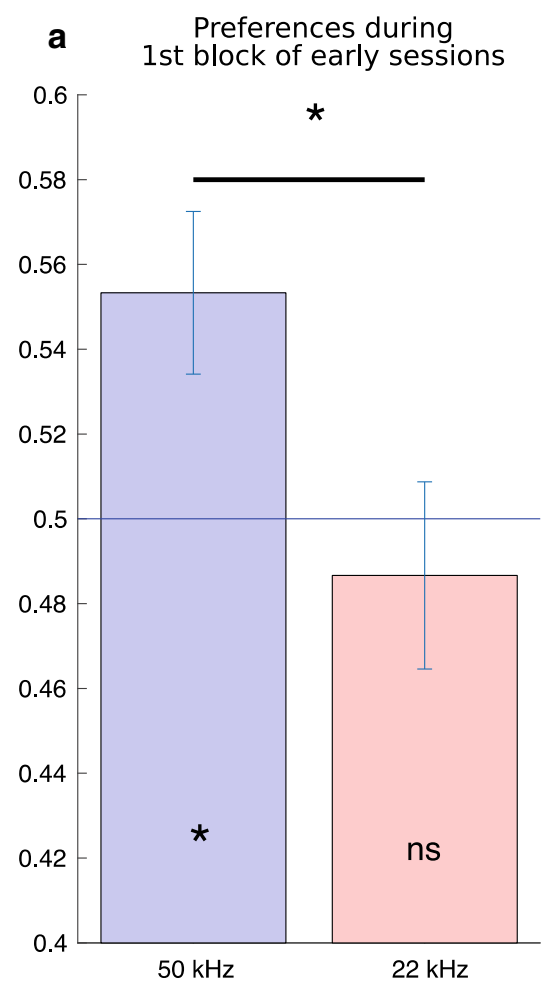

\section{b USV Stimuli of different origin}

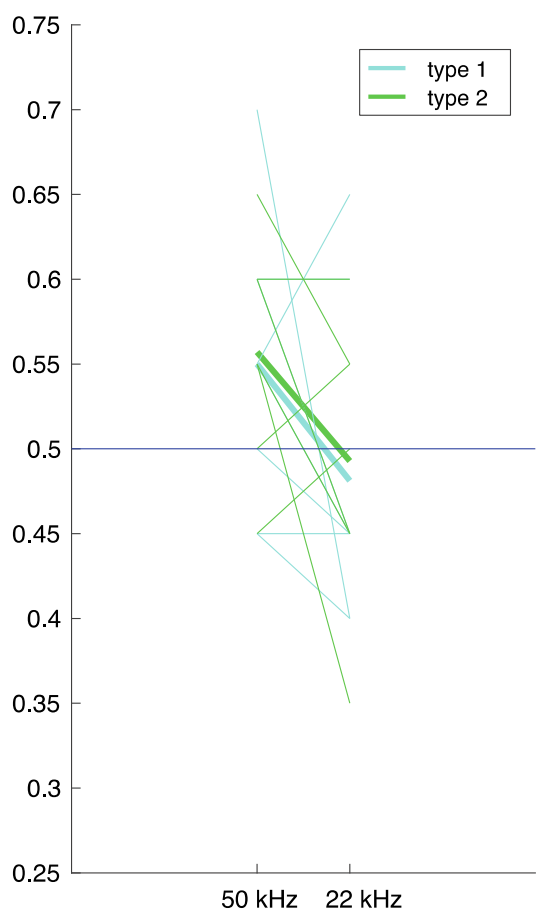

Fig. 6 Preferences in 50-vs-Noise and 22-vs-Noise sessions, averaged across rats for the first block of five trials (1-5) and the first half of sessions (1-4). a Preference for 50 over noise was significantly above chance, while no significant difference from chance could be detected in the 22-vs-Noise sessions. The difference in preference for both session types was significant, though. b Individual data points for the data in a, now also split by stimulus type. No difference between stimulus type 1 (blue) and stimulus type 2 (green) could be detected

reinforcers, and that the probability of repeating actions associated with $22-\mathrm{kHz}$ USVs is lower relative to $50-\mathrm{kHz}$ USVs or non-ultrasonic control stimuli. Using an experimental paradigm adapted from the rodent PCT, we confirmed the reinforcing quality of USV playback, most prominent in the preference exhibited by rats for the playback of appetitive $50 \mathrm{kHz}$ USV calls over control acoustic stimuli. The reinforcing quality is transient, however, as predicted from the literature (see below). Finally, we used two different sets of stimuli to test our hypothesis: one set of USVs was recorded from Wistar rats (Wöhr \& Schwarting, 2007) and the other set from Long-Evans rats, as described above. We found that Long-Evans rats did not respond differently to USVs originating from conspecific Long-Evans rats, or from a different strain-Wistar rats.

Previous studies showed that $50-\mathrm{kHz}$ USV stimuli induce strong, but transient approach behavior during initial playback and that this approach response quickly attenuated across trials (Wöhr \& Schwarting, 2012; Seffer et al., 2014), together with 
a decline in physiological measures of the rewarding properties of the USV stimulus (Willuhn et al., 2014). The authors explained this effect by USVs being secondary reinforcers that, after repeated exposure might, at least partially, lose their value (Willuhn et al., 2014). This explanation is in line with our hypothesis that USVs are not rewarding or aversive by themselves, but only by their virtue of carrying social significance in a social context.

A further issue that warrants elaboration is the nature of the motivating property of the USV stimulation. Because the USV playback stimuli were consistently paired with food rewards, as was the case in the partner session in the rodent PCT, we cannot conclude with certainty that USV playback by itself motived approach or avoidance behavior in the present study. Rather, the USV stimuli might have modulated the reinforcing value of the food rewards; that is the appetitive value of the food rewards was possibly enhanced by pairing it with $50-\mathrm{kHz}$ playback and it was possibly reduced by pairing it with $22-\mathrm{kHz}$ playback. Such a putatively modulating, rather than activating, effect of the USV stimuli on motivation might explain the relatively mild and transient size of the effects reported here.

Finally, our Long-Evans rats showed identical behavior towards USV stimuli recorded from Wistar and conspecific Long-Evans rats. Taken together, these data support our hypothesis that 50-kHz USVs, in contrast to comparable, but non-social acoustic stimuli, act as positive social reinforcers that influence behavior and might, therefore, contribute to orchestrating social interaction between rats. Our findings corroborate and extend the results of a recent study that showed that rats show instrumental responses to produce $50-\mathrm{kHz}$ USV playback in a non-spatial operant conditioning setup (Burgdorf et al., 2008). However, the evidence for a putative role of 22-kHz USVs as negative social reinforcers is less conclusive. This result suggests that positive, rather than negative social feedback might drive the spatial preferences linked to different social outcomes (partner also rewarded or not) in the pro-social choice task.

Although the social reinforcement mechanism described here and elsewhere (Hernandez-Lallement, van Wingerden, et al., 2017) provides a parsimonious, plausible and realistic explanation for rat social behavior, it is agnostic about how rats actually cognitively represent their social world: as discussed above, our social reinforcement theory does not explain how a rat conceptually links and represents the different stimulus levels - the USV's physical dimension (rhythmic oscillations of air compression and deflation creating auditory perception), the emotional level (the putative enjoyment or averseness of listening to USVs) and their motivational level (50 kHzUSVs are wanted and they prompt action to obtain them) - into a coherent cognitive representation of a social situation. In the following section, we will present a philosophically inspired attempt to theoretically model how rats link and process these stimulus levels into a complex cognitive representation of social interaction.

The second part of this paper, thus, attempts to provide a novel approach to animal learning and cognition. The "cascade" approach regards the categorization and cognitive representation of types of action as potentially multilevel. When a rat learns in an experimental setting that certain types of action are rewarding, its brain is assumed to form an action cascade that categorizes this type of action simultaneously 
as an act of getting a reward. The multilevel approach can be applied to model social behavior as multilevel: a cognitive complex of performing the basic physical behavior and thereby at the same time a particular kind of social behavior. Applying likewise to human cognition, cascade theory is a candidate for connecting animal and human cognition.

\section{Part II: Cascades in Animal Cognition}

\section{A Cognitive Perspective: Acting at Multiple Levels}

This section offers a theoretical perspective on the neurocognition of the representation of action. Applied to rats, it is not to be taken as a theory rival to existing psychological accounts of animal learning, but rather as an account concerning the cognitive representation involved and the cognitive implementation of conditioning. The most prominent feature of the "Cascade" theory of cognitive representation is a multilevel approach to categorization. It applies, it appears, to humans and animals likewise. ${ }^{1}$

\subsection{Goldman's Multilevel Theory of Human Action}

\subsubsection{Goldman's Notion of Level-Generation and the Notion of Cascade}

When humans categorize and conceptualize an action, they usually do it in more than one way at the same time. The philosopher Alvin Goldman developed a theory of human action that is based on this principal observation (Goldman, 1970). If I open a door, this is a physical act of interaction with an object that changes its state. Opening a particular door can be achieved by a variety of bodily actions. If it is a hinged door, I can push the door at its handle or somewhere else with my hand, I can push it with my foot, I can lean against it with my shoulder or my back; depending on my position and the construction of the door, I may have to pull at the door. For sliding doors or automatic doors, other types of action are required. Thus, 'opening a door' refers to at least two levels of action: (1) the basic physical action one applies to the door, and (2) the more abstract functional level of causing the door to open. The acts at the physical and at the functional level do not concern the same properties of the door. The physical act changes the spatial position of the door leaf or leaves. The higher-level act concerns states of the door that are related to its functioning as an object that is used to obstruct or enable access to a space behind it.

The lower-level action is necessary for achieving the higher-level action. This achievement is not automatic but requires certain circumstances; for example, the

\footnotetext{
${ }^{1}$ The theory is introduced in more depth and detail in Löbner (this volume).
} 
mechanical door must not be locked, the automatic door must be in function. Goldman (1970) speaks of "level-generation" if actions are related in this way: under certain circumstances, the lower-level action "generates" the higher-level action, the lowerlevel action is a method of doing the higher-level thing; by pushing the door or pulling at it, one opens it. While in this case the level-generating relation is based on causation, there are also other mechanisms such as conventional level-generation; for example, if I nod my head, this may conventionally generate an approval or permission because nodding one's head is a conventionally established method of approving or permitting.

Crucially, if an action A generates a higher-level action B, A and B are actions by the same agent and at the same time, done in one. It is very important to note that level-generation does not relate an action to an event it causes. If I open the door for someone and let them pass, I first open the door and then the other will pass through the door a moment later. Level-generation does not obtain between these consecutive actions by two different agents. Rather it obtains between the action of opening the door and the action of opening a passage for the other. These two actions are actions by the same agent and they occur at strictly the same time. It is this feature of Goldman's theory of action that makes it a theory of multilevel categorization.

According to Goldman, a basic action may level-generate more than just one higher-level action; it can generate a complex multilevel structure of actions with many steps that build on each other; the structure can also branch into different lines of generation. For example, by pushing a door and opening it, one may at the same time open a passage in an aisle as well as cause an air draft; opening the passage may in turn generate doing a favor; causing a draft may further generate making a window slam. We will give complex examples below. Goldman uses the term "act-trees" for structures created by level-generation; we prefer to call them "cascades" as there are good reasons to transfer the notion to other things than action ${ }^{2}$. Crucially, the actions that form a cascade are actions of different type. For example, leaning against the door and opening the door are not actions of the same type. A door can be opened by other methods, and leaning against the door can have other effects than opening it; for example, it may as well be an act of closing the door, or of keeping the door closed if somebody is pushing against it from the other side.

\subsubsection{Goldman's Level-Theory as a Psychological Theory of Categorization}

It is convenient to use the term 'doing' for that to which a cascade description applies: there is one doing, for example with the door, but this one doing can be categorized in many different ways as constituting as many different types, or categories, of action as the cascade provides. In the discussion of his theory of action with other philosophers, Goldman emphasizes that the distinction of types involved in a cascade of action is a psychological distinction, not a distinction of things out there in the

${ }^{2}$ See Löbner, this volume, ss. 5-7. 
world. The cascade agent produces one doing, but it is categorized simultaneously at different levels in a hierarchy of level-generation (Goldman, 1979). A cascade forms in our minds, in our view and cognitive modeling of what is going on or what we are doing ourselves. What a person does in a concrete situation, to us, is, in our reality, all these acts in the cascade at the same time. The particular doing in our door example of level-generation may belong at the same time to the action categories 'push against the door', 'open the door for Adam', 'do Adam a favor', and maybe others. It is important to realize that the different categories we may apply to the one underlying doing are not just a bunch of categories that are somehow associated. Rather they are organized in a tree structure of dependence. The higher actions depend for their coming about on the lower actions that "generate" them. And all higher-level actions depend on necessary circumstances to come about.

As the door example illustrates, the formation of cascades takes place even with as simple actions as opening a door. We may well assume that humans categorize almost any willful action by a human as a cascade of action rather than just as the basic physical doing. We will inevitably try to interpret the actions of others in terms of the intentions they pursue by doing what they do; if they act on an artefact in a normal way, for example on a door, we will assume that the action is related to the usual function of the object. Thus, categorizing an action as 'opening the door' would provide a causal explanation of the observable physical act.

\subsubsection{Social Action and Interaction}

One observation relevant in our context is the fact that social action necessarily constitutes higher-level action. Searle (1995) developed a theory of social reality that distinguishes between a physical level and a social level of action, persons, and objects. A certain movement with the head is an approval if and only if it counts as such; a human is the president of Canada if and only if they count as such, and a piece of paper is money if and only if it counts as money. The things that count as something in these examples are physical entities and what they count as are social, entities that is, entities in our social reality. Notably, in all these cases, the things considered are necessarily both at the same time: the physical entity and the socialreality entity. For the part of Searle's theory concerning acts, the relation between things at the physical and at the social level is captured by Goldman's more general notion of level-generation.

As a consequence of the principal higher-level character of social action, social behavior always 'parasitizes' on more basic physical behavior. ${ }^{3}$ For example, one may turn up the corners of one's mouth and expose the front teeth and thereby levelgenerate a smile which, if directed at someone, may under circumstances constitute a social signal which constitutes a display of affection, or something else. Up from the level 'smiling at someone', the cascade reaches a social level. If we go back to

\footnotetext{
${ }^{3}$ The terminus parasite was introduced in this connection by Kearns (2003) who relates to the lower and the higher level of a two-level cascade as 'host' and 'parasite', respectively.
} 
Fig. 7 A door-opening cascade: doing six things in one

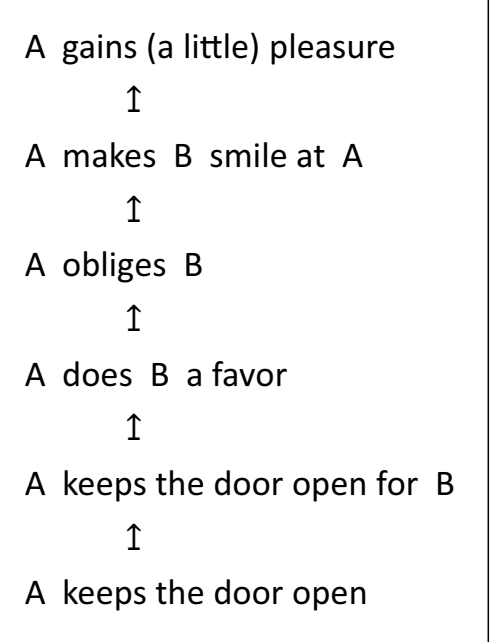

the example in the introduction, we get an even more complex structure. Using an upward arrow $\uparrow$ for level-generation, we can represent the cascade bottom-up as in Fig. 7.

As the example illustrates, own action may cascade to ultimately giving oneself a pleasure (or any other kind of emotional experience) by doing what one does. Obviously, this too cannot be done without the support of some physical action. We may keep in mind two general points about cascades: (i) physical action may cascade to social action, and (ii) action may cascade to obtaining an emotional experience, where emotional-level action may or may not come about by means of social-level action like in our fictitious example.

There is another aspect to the door-opening example. Social reality is constructed interactively (see Clark (1996) on a multilevel interactional account of verbal communication). If $\mathrm{A}$ keeps a door open for $\mathrm{B}$, meaning to do $\mathrm{B}$ a favor and cascading the conceptualization of their own act correspondingly, then the thanks A receives from $B$ will confirm that A and B share the social construal of their interaction: B would not have thanked A if B had not construed A's act as involving the level-generation of doing B a favor. An analogous consideration applies to the next level above the favor: the level-generation of obliging B by doing B a favor. Acknowledgment and confirmation of this additional, emotional level, is executed by B sending a smile to A. Given that receiving a smile is felt as something pleasant, the level-generation of 'B please A' by 'B smile at A' is part of the joint construal of B's reaction. 


\subsection{Cascades and Learning}

Goldman's theory was constructed for the categorization of individual action tokens. It can, however, also be applied to the consistent multilevel categorization of recurring types of action. For example, if we experience that the light goes on when we flip a certain switch, and if we repeat the action and achieve the same effect, we will learn a cascade: that flipping this switch goes with switching that light on. We acquire a piece of procedural knowledge by memorizing a two-level action cascade concept composed of the two single-level action concepts 'flip this switch' and 'turn on that light'. Our environment being as it is, we will easily generalize this cascade to other switches and other lights, and so on. Thus, action learning is cascade learning, at least for all but merely physical basic action like turning one's head or lifting ones hand. We learn that doing one thing also means doing the higher-level thing, and the level above that, and so on. An action and the higher level achieved with it are conflated into one concept. Cascade learning may also include that, by an action, we trigger approval or disapproval, cause pleasure or pain, a particular taste or other bodily sensations. If we assume that cascading plays a role in concept formation, we may conclude that action concepts are formed that link basic actions and the recurring achievement of certain causal effects into one multilevel concept.

It is important to note that even for humans, kids or adults, learning of action cascades does not necessarily involve reflection. It just requires that the learning subject register that the lower-level action goes with the higher-level action. In particular, the learning of cascade levels that are causally linked does not require any causal understanding. We learn that pressing the red button of the TV remote control means turning the TV on or off, but we may well die without ever having understood what we actually do at the technical level by pressing this button. This level of understanding is not relevant for learning how to succeed in dealing with TVs and remote controls. To know how to deal with a remote control is essentially 'knowledge how' ${ }^{4}$, and the mechanism by which we acquire this knowledge is learning by doing.

Cascade learning does not only concern practical abilities. A child may cascadelearn that a certain kind of behavior always upsets her mother; the child will register this and adjust her behavior accordingly, but may possibly never understand why her mother reacted that way. We learn in countless regards that our actions are accompanied by higher cascade levels of particular qualities. Cascade learning will result in a "practical" implicit understanding of the environment, in the sense that we learn which intended or unintended higher-level kinds of action are generated by certain other kinds of action. We learn things like "if I do x, I give myself experience $y$ ". Given that we are able to undertake certain action or refrain from it, this kind of understanding our environment will enable us to adapt to it.

Cascade knowledge need not be accessible to consciousness: we may have it without being aware of it and without being able to describe it. For example, pronouncing a word in a way that enables others to recognize it phonetically means to enact a cascade of production based on intentional action of our articulatory organs

\footnotetext{
${ }^{4}$ See Katzoff (1984) for the connection of knowing how to Goldman's theory of action.
} 
to produce articulated sounds, thereby producing speech sounds, thereby producing certain speech phonemes, and with them an established sound form of a word in a particular language. All this is stored in the language production repertoire-a normal language user is not aware of the levels of actions involved and they would not be able to describe what they do at which levels. All they need to be able to do is to aim at doing something particular at a pretty high level, something with the result of making audible a particular sound pattern.

\subsection{Applying Cascade Theory to Rat Behavior in the Experiments Reported}

We proceed to propose that the cascade model of action categorization and action learning applies to rats as well. First of all, it appears that there are certain types of rat action that are relevant to the actors at levels beyond the mere physical doing. Among these are levels that constitute social action. For example, if young rats do rough-and-tumble play, they recognize that this is not hostile fight: crucially, the fight is 'friendly' to both of them. In some way or other, they succeed in letting the other "know" that their own behavior is not hostile, and they succeed in categorizing the other's behavior in the same way. Both rats engaged in a rough-and-tumble play possess two categorizations of representing physical fight or else fight-like action. At a lower level they categorize the physical action, at a higher, social, level they categorize it as hostile fighting or as play. At the lower level they "know" bodily methods of fighting, for instance, pushing or biting, and they are able to modulate these methods as to cascade either to a real fight or to rough-and-tumble play. There can be no doubt that a rough-and-tumble play to the rats is both, bodily interaction and a social interaction that is different from hostile fight. What they do has a function to them at both levels, as some sort of bodily learning and some sort of social learning.

When we say that this "is to the rats" a particular type of action, we do not imply consciousness on the part of the rat. The cascade view does not commit us to the assumption that rats have consciousness (at least not in the same sense as humans); it only commits us to assume that the rats' brains categorize the rats' doing in these ways and that, in this sense, the rats register what is going on at both levels. As rats are able to recognize and repeat types of action, for example under experimental conditions, they must have cognitive representations of types of action. Crucially, they register the character of what is going on not only for their own part, but also for the part of their interaction partner.

Among the actions that have multilevel character to rats are the USVs (ultrasonic vocalizations) mentioned above. The fact that these vocalizations trigger brain reactions associated with emotion, shows that these are not just plain sound productions (like, for example, the production of the sound they produce when they scratch their ear or shuffle around); these special sound productions are 'received' at an 
acoustic and an emotional level. We do not know if the rat, when hearing a 50$\mathrm{kHz}$ USV, hears this as a display of comfort or pleasure. If this should be the case, the rat might have a two-level representation of the act by their conspecific. All we seem to be entitled to assume at present is that $50-\mathrm{kHz}$ USVs must have a pleasant 'ring' to the perceiver. But this is sufficient for the assumption of a two-level neural cascade representation of $50-\mathrm{kHz}$ USVs issued by other rats, whence these USV's carry emotional significance.

In the experiments, the rats learn. They acquire behavior. The experiments are designed in the way that the behavior acquired leads to getting themselves a reward. We can apply the cascade model to the learning process, if we assume that learning a particular behavior consists in acquiring a multilevel action cascade. The general structure of reward-inforced learning would be the acquisition of a cascade that amounts to: 'do x' 1 'get a reward'; here 'get' is to be taken to mean active acquisition, not just passive reception, because the latter would not be an action by the animal.

Assume that the actor rat learns that it will receive pellets upon entering compartment $\mathrm{c} 1$. That learnt, the rat will repeat the action if it likes to get pellets. This behavior can be interpreted as involving the acquisition of an action cascade of three levels:

$\begin{array}{cc}\text { [Level 3] } & \text { get pleasure } \\ & \uparrow \\ \text { [Level 2] } & \text { get pellets } \\ & \uparrow\end{array}$

[Level 1] enter compartment c1

One might speculate that it is the rewarding course of events that supports not just the behavior as such, but primarily the formation of the cascade described; if the animal forms and then memorizes the cascade, this results in a mental condition that enables the animal to repeat these rewarding experiences at will by taking the action at the bottom of the cascade.

In the prosocial choice task experiments described in Hernandez-Lallement et al. (2015), some rats seem to have learnt just Cascade 1. The prosocial rats, however, developed a behavior that involves a more complex cascade structure with a second branch on the first node (Fig. 8, blue branch).

They register that the partner rat gets pellets, too, and their brain ascribes it to themselves as a generated higher-level action. As for the third step of the cascade, we know that there are $50 \mathrm{kHz}$ USVs when the actor rat and the partner rat simultaneously get their pellets; however, due to the technical equipment used, it was not possible to ascribe the vocalizations to one or the other rat or to both. We are entitled to assume that the actor rat sees and thereby registers that the partner rat gets pellets. We do not know whether this constitutes a pleasant experience to the actor rat. If we could be sure that the partner rat produces a $50 \mathrm{kHz}$ USV, we might assume that the actor rat hears it and experiences this as an emotional reward. We can explain the preference for this condition only if we assume that the prosocial behavior cascades 
Fig. 8 Act-trees for the actions taken by the rats in the PCT experiment. The black branch represents the action cascade acquired by every rat in this experiment. The blue structure represents the additional cascade branch acquired by the rats that develop prosocial behavior

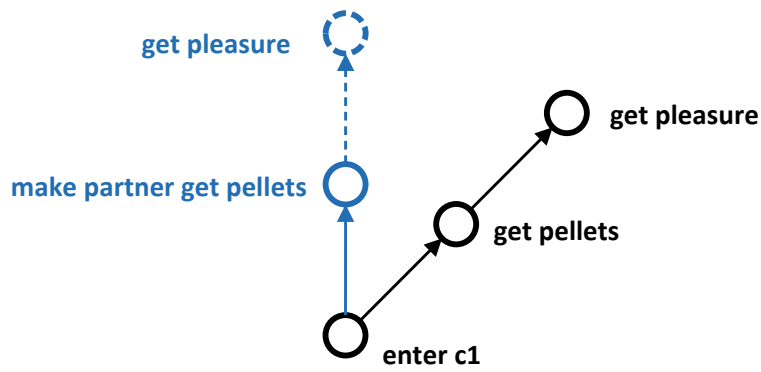

to an additional reward in the left branch of the cascade. The left cascade branch would then level-generate an additional third-level 'get pleasure'.

In the new experiments described above, with no partner rat present, the USV constitutes an additional two-step branch generated by Level 2 in the first cascade, to be construed as 'get a $50 \mathrm{kHzUSV}$ ', thereby 'get pleasure'. We will assume that the two-way reward (transiently) outweighs the one-way reward of the no-USV condition. An explanation as to why the effect of the USV gets weaker in the course of the experiment will not be attempted here.

\subsection{Psychological Commitments of the Cascade Approach}

Application of the cascade model to rat learning involves certain psychological commitments.

(i) The rat's brain implements cascade formation.

The rat's brain creates links between basic types of bodily action and what goes with them, perceptibly to the rat; if the rat brain works in this way, it ascribes the effects of behavior to the behavior itself, connecting, for example, eating certain food to staying hunger. In this way, the animal learns by experience what its behavior "means" to it. When we talk of "meaning" here, we mean it in the basic sense of immediate concomitance, not involving reasoning or convention: if something is of category A and category A cascades to category B, then this instance of A "means"/"constitutes"/simply "is" also an instance of B-to the cognitive subject.

Level-generation presupposes that the animal perceives its own action, and attributes it to itself. This results in a second psychological commitment:

(ii) Rats have a (weak) sense of agency. Their brain records their action.

There can be no doubt that rats, by way of proprioception and perception of the environment, sense that they are acting.

In addition, we need to assume that the rat's brain categorizes what the animal is doing. This amounts to the following commitment:

(iii) The rat's brain forms concepts (representations) of types of own action. 
Crucial for the cascade formation is the following assumption:

(iv) The rat's brain assigns credit to the animal itself for what happens concomitant with action of the particular type.

Cascade formation then means that the rat's brain generates a higher cascade level for the underlying action concept that amounts to making happen what happens after action of the given type.

There are restrictions on this condition. First, we will assume that it holds only for such events following the rat's action that are significant to the animal's well-being, and hence of "interest". Second, there is supposed to be a limit on the time that may lapse between the rat's action and later events. The rat's brain will possibly not connect the animal's doing to things that happen after a long time.

It appears that commitments (ii) to (iv) are uncontroversial; we construe the changes in behavior of rats in experimental settings as learning behavior under the conditions of the experiment. This would be unexplainable if we would not assume that the animals' brains register the animals' doings as their own and as of a particular type and if their brains did not credit the animals with what follows their own action as something they can ascribe to this type of action. ${ }^{5}$

(v) The rat's brain stores in long-term memory the repeated concomitance of certain effects with a type of own action.

This means that the rat's brain connects this type of action-not only individual single action tokens - with this kind or result.

Of course, the crucial assumption is the first one. The other assumptions are implicit in everyday experimental practice.

\subsection{What Can the Cascade Approach Buy Us?}

What the cascade theory buys us is twofold. First, it provides a fundamental neurocognitive mechanism for a model of the animal's learning about its environment. If the animal's brain builds cascades on the types of physical action the animal is capable of, then the brain integrates the type of action with the achievement of its results into one multilevel concept. The type of action is thereby invested with a particular significance for the animal, for example emotional significance, significance relevant for survival, or the significance of performing a certain type of social action or interaction. Cascade-format action concepts link an action to the achievement of its result as something ascribed to the animal as self-caused - and thereby controlled by own behavior. Cascade learning of effects of their doing invests the animals with the ability to choose ways of action, to seek advantage and avoid disadvantage. Thus, cascade formation for own-action types provides a basic mental mechanism of adapting to the environment, including the animal's social group, in a learning-by-doing way.

\footnotetext{
${ }^{5}$ See Takahashi et al. (2011) for exceptional conditions of the animals under which the credit assignment required does not work.
} 
Second, the cascade approach offers an explanation for the way in which an animal is able to acquire a practical understanding of the ways of its environment, as its brain links types of behavior to the triggering of its outcome. If the cognitive system of an animal is equipped with the ability of action cascade formation-i.e. if it records what the animal does to itself if it acts in this way or another-it enables adaptation to the environment without requiring any level of causal understanding, reasoning, or modeling. Thus, the cascade model of learning is a model of learning by doing and what is acquired is plain knowledge-how.

The cascade approach might be successful in modeling multilevel categorization across humans and animals, in particular as part of modeling the acquisition of multilevel action concepts and methods of how to do things, and of what is "social reality' to the cognitive subjects. Another way of looking at cascade theory is to consider it a psychological theory of "meaning", in the very basic sense that acting at a lower cascade level also "means" to act at the generated higher level. In this sense, cascading provides action with meaning to the cognitive subject.

In the field of cognitive theory and psychology, the theory is at its very beginning. It seems to be able to claim some plausibility (cf. Vallacher \& Wegner, 2011). In any event it would be interesting to try to develop methods for testing it experimentally. For example, a cascade approach to learning raises concrete questions concerning structural constraints on cascades to be acquired in terms of the number of levels, of branching complexity, and of memorizability.

\section{Conclusions}

In this study, we present evidence supporting our hypothesis that USVs act as social reinforcers. In line with the social reinforcement hypothesis (Hernandez-Lallement, van Wingerden, et al., 2017), we show that rats preferred T-maze compartments associated with 50-kHz USV playback over compartments associated with non-ultrasonic control stimuli. This observation fuels the hypothesis that USVs might orchestrate and structure social interaction between rats. Finally, we argue that one avenue towards understanding the conceptual representation of the emotional and motivational significance of rat USVs might require a multilevel approach, as proposed by Goldman (1970) in his cascade model of mental representation of human action.

Acknowledgements This work was supported by the German Research Foundation (Deutsche Forschungsgemeinschaft), projects B09 and D03 of CRC 991 "The structure of representations in language, cognition, and science". Mireille van Berkel is supported by Volkswagen Freigeist fellowship no. AZ88216. Maurice-Philipp Zech is supported by a grant from the German Research Foundation (grant no. KA 2675/5-3). 


\section{References}

Ben-Ami Bartal, I., Decety, J., \& Mason, P. (2011). Empathy and pro-social behavior in rats. Science, 334, 1427-1430.

Blanchard, D. C., \& Blanchard, R. J. (1990). Behavioral correlates of chronic dominancesubordination relationships of male rats in a seminatural situation. Neuroscience and Biobehavioral Reviews, 14, 455-462.

Blanchard, R. J., Flannelly, K. J., \& Blanchard, D. C. (1988). Life-span studies of dominance and aggression in established colonies of laboratory rats. Physiology \& Behavior, 43(1), 1-7.

Brudzynski, S. M. (2013). Ethotransmission: communication of emotional states through ultrasonic vocalization in rats. Current Opinion in Neurobiology, 23(3), 310-317.

Brudzynski, S. M., \& Ociepa, D. (1992). Ultrasonic vocalization of laboratory rats in response to handling and touch. Physiology \& Behavior, 52(4), 655-660.

Burgdorf, J., Knutson, B., Panksepp, J., \& Ikemoto, S. (2001). Nucleus accumbens amphetamine microinjections unconditionally elicit $50-\mathrm{kHz}$ ultrasonic vocalizations in rats. Behavioral Neuroscience, 115(4), 940-944.

Burgdorf, J., Kroes, R. A., Moskal, J. R., Pfaus, J. G., Brudzynski, S. M., \& Panksepp, J. (2008). Ultrasonic vocalizations of rats (Rattus norvegicus) during mating, play, and aggression: Behavioral concomitants, relationship to reward, and self-administration of playback. Journal of Comparative Psychology, 122(4), 357-367.

Calvino, B., Besson, J. M., Boehrer, A., \& Depaulis, A. (1996). Ultrasonic vocalization (22-28 kHz) in a model of chronic pain, the arthritic rat: effects of analgesic drugs. NeuroReport, 7(2), 581-584.

Chang, S. W., Fagan, N. A., Toda, K., Utevsky, A. V., Pearson, J. M., \& Platt, M. L. (2015). Neural mechanisms of social decision-making in the primate amygdala. Proceedings of the National Academy of Sciences, 112(52), 16012-16017.

Clark, H. H. (1996). Using Language. Cambridge University Press.

Engelhardt, K. A., Fuchs, E., Schwarting, R. K., \& Wöhr, M. (2017). Effects of amphetamine on pro-social ultrasonic communication in juvenile rats: Implications for mania models. European Neuropsychopharmacology, 27(3), 261-273.

Fehr, E., \& Fischbacher, U. (2003). The nature of human altruism. Nature, 425(6960), 785-791.

Fehr, E., \& Schmidt, K. M. (1999). A theory of fairness, competition and cooperation. The Quarterly Journal of Economics, 114, 817-868.

Fiske, S. T., \& Taylor, S. E. (1984). Social Cognition. Reading and MA: Addison-Wesley Publishing. Goldman, A. I. (1970). Theory of Human Action. Princeton, New Jersey: Princeton University Press. Goldman, A. I. (1979). Action, Causation, and Unity. Nous, 13, 261-270.

Hernandez-Lallement, J., van Wingerden, M., Marx, C., Srejic, M., \& Kalenscher, T. (2015). Rats prefer mutual rewards in a prosocial choice task. Frontiers in Neuroscience, 8, 443.

Hernandez-Lallement, J., van Wingerden, M., Schäble, S., \& Kalenscher, T. (2016). Basolateral amygdala lesions abolish mutual reward preferences in rats. Neurobiology of Learning and Memory, 127, 1-9.

Hernandez-Lallement, J., van Wingerden, M., \& Kalenscher, T. (2017). Towards an animal model of callousness. Neuroscience and Biobehavioral Reviews.

Hernandez-Lallement, J., van Wingerden, M., Schäble, S., \& Kalenscher, T. (2017b). A social reinforcement learning hypothesis of mutual reward preferences in rats. Current Topics in Behavioral Neurosciences, 30, 159-176.

Ishiyama, S., \& Brecht, M. (2016). Neural correlates of ticklishness in the rat somatosensory cortex. Science, 354(6313), 757-760.

Janak, P. H., \& Tye, K. M. (2015). From circuits to behaviour in the amygdala. Nature, 517(7534), 284-292.

Kalenscher, T., \& van Wingerden, M. (2011). Why we should use animals to study economic decision making: A perspective. Frontiers in Neuroscience, 5(82), 1-11. 
Kashtelyan, V., Lichtenberg, N. T., Chen, M. L., Cheer, J. F., \& Roesch, M. R. (2014). Observation of reward delivery to a conspecific modulates dopamine release in ventral striatum. Current Biology, 24(21), 2564-2568.

Katzoff, C. (1984). Knowing how. The Southern Journal of Philosophy, 22, 61-68.

Kearns, K. (2003). Durative achievements and individual-level predicates on events. Linguistics and Philosophy, 26(5), 595-635.

Knutson, B., Burgdorf, J., \& Panksepp, J. (1998). Anticipation of play elicits high-frequency ultrasonic vocalizations in young rats. Journal of Comparative Psychology, 112(1), 65-73.

LeDoux, J. E. (1994). Emotion, memory and the brain. Scientific American, 270(6), 50-57.

LeDoux, J. E., Cicchetti, P., Xagoraris, A., \& Romanski, L. M. (1990). The lateral amygdaloid nucleus: sensory interface of the amygdala in fear conditioning. Journal of Neuroscience, 10(4), $1062-1069$.

Löbner, S. (this volume). Cascades. Goldman's level-generation, multilevel categorization of action, and multilevel verb semantics.

Lukas, M., \& Wöhr, M. (2015). Endogenous vasopressin, innate anxiety, and the emission of pro-social $50-\mathrm{kHz}$ ultrasonic vocalizations during social play behavior in juvenile rats. Psychoneuroendocrinology, 56, 35-44.

Oberliessen, L., Hernandez-Lallement, J., Schäble, S., van Wingerden, M., Seinstra, M., \& Kalenscher, T. (2016). Inequity aversion in rats, Rattus norvegicus. Animal Behaviour, 115, $157-166$.

Oostenveld, R., Fries, P., Maris, E., \& Schoffelen, J. M. (2011). FieldTrip: Open source software for advanced analysis of MEG, EEG, and invasive electrophysiological data. Computational Intelligence and Neuroscience, 2011, 156869, 9. https://doi.org/10.1155/2011/156869.

Panksepp, J., \& Burgdorf, J. (2000). 50-kHz chirping (laughter?) in response to conditioned and unconditioned tickle-induced reward in rats: Effects of social housing and genetic variables. Behavioural Brain Research, 115(1), 25-38.

Parkinson, J. A., Robbins, T. W., \& Everitt, B. J. (1996). Lesions of the nucleus accumbens core, but not basolateral amygdala or subiculum, disrupt stimulus-reward learning in a novel autoshaping procedure. Paper presented at the Society for Neuroscience Abstracts.

Parsana, A. J., Li, N., \& Brown, T. H. (2012). Positive and negative ultrasonic social signals elicit opposing firing patterns in rat amygdala. Behavioral Brain Research, 226(1), 77-86.

Rutte, C., \& Taborsky, M. (2007). Generalized reciprocity in rats. PLoS Biology, 5(7), e196.

Sadananda, M., Wöhr, M., \& Schwarting, R. K. (2008). Playback of 22-kHz and 50-kHz ultrasonic vocalizations induces differential c-fos expression in rat brain. Neuroscience Letters, 435(1), $17-23$

Sales, G. D. (1972). Ultrasound and aggressive behaviour in rats and other small mammals. Animal Behaviour, 20(1), 88-100.

Searle, J. R. (1995). The construction of social reality. New York: The Free Press.

Seffer, D., Schwarting, R. K., \& Wöhr, M. (2014). Pro-social ultrasonic communication in rats: Insights from playback studies. Journal of Neuroscience Methods, 234, 73-81.

Siviy, S. M., \& Panksepp, J. (2011). In search of the neurobiological substrates for social playfulness in mammalian brains. Neuroscience and Biobehavioral Reviews, 35(9), 1821-1830.

Strombach, T., Weber, B., Hangebrauk, Z., Kenning, P., Karipidis, I. I., Tobler, P. N., et al. (2015). Social discounting involves modulation of neural value signals by temporoparietal junction. Proceedings of the National Academy of Sciences, 112(5), 1619-1624.

Sutton, R. S., \& Barto, A. G. (2012). Reinforcement Learning: An Introduction. Cambridge, Massachusetts: The MIT Press.

Takahashi, Y. K., Roesch, M. R., Wilson, R. C., Toreson, K., O’Donnell, P., Niv, Y., et al. (2011). Expectancy-related changes in firing of dopamine neurons depend on orbitofrontal cortex. Nature Neuroscience, 14(12), 1590-1597.

Vallacher, R. R., \& Wegner, D. M. (2011). Action identification theory. In P. Van Lange, A. W. Kruglanski, \& E. T. Higgins (Eds.), Handbook of theories in social psychology (pp. 349-370) London, England: Sage. 
Vanderschuren, L. J., Achterberg, M. E., \& Trezza, V. (2016). The neurobiology of social play and its rewarding value in rats. Neuroscience and Biobehavioral Reviews, 70, 86-105.

Willuhn, I., Tose, A., Wanat, M. J., Hart, A. S., Hollon, N. G., Phillips, P. E., et al. (2014). Phasic dopamine release in the nucleus accumbens in response to pro-social $50 \mathrm{kHz}$ ultrasonic vocalizations in rats. Journal of Neuroscience, 34(32), 10616-10623.

Wöhr, M., \& Schwarting, R. K. (2007). Ultrasonic communication in rats: can playback of 50-kHz calls induce approach behavior? PLoS ONE, 2(12), e1365.

Wöhr, M., \& Schwarting, R. K. (2012). Testing social acoustic memory in rats: effects of stimulus configuration and long-term memory on the induction of social approach behavior by appetitive 50-kHz ultrasonic vocalizations. Neurobiology of Learning and Memory, 98(2), 154-164.

Wöhr, M., \& Schwarting, R. K. (2013). Affective communication in rodents: ultrasonic vocalizations as a tool for research on emotion and motivation. Cell and Tissue Research, 354(1), 81-97.

Open Access This chapter is licensed under the terms of the Creative Commons Attribution 4.0 International License (http://creativecommons.org/licenses/by/4.0/), which permits use, sharing, adaptation, distribution and reproduction in any medium or format, as long as you give appropriate credit to the original author(s) and the source, provide a link to the Creative Commons license and indicate if changes were made.

The images or other third party material in this chapter are included in the chapter's Creative Commons license, unless indicated otherwise in a credit line to the material. If material is not included in the chapter's Creative Commons license and your intended use is not permitted by statutory regulation or exceeds the permitted use, you will need to obtain permission directly from the copyright holder.

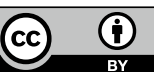

